\title{
Clinical Study \\ Selective Laser Trabeculoplasty for Glaucoma Secondary to Emulsified Silicone Oil after Pars Plana Vitrectomy: A Pilot Study
}

\author{
Zeynep Alkin, ${ }^{1}$ Banu Satana, ${ }^{1}$ Abdullah Ozkaya, ${ }^{1}$ Berna Basarir, ${ }^{1}$ Cigdem Altan, \\ Ahmet Taylan Yazici, ${ }^{1}$ and Ahmet Demirok ${ }^{1,2}$ \\ ${ }^{1}$ Beyoglu Eye Training and Research Hospital, Bereketzade Cami Sokak, No. 2, Beyoglu, 34421 Istanbul, Turkey \\ ${ }^{2}$ Medeniyet University, Dumlupinar Mahallesi, D-100 Karayolu, No. 98, Kadikoy, 34270 Istanbul, Turkey \\ Correspondence should be addressed to Zeynep Alkin; zeynepalkin@gmail.com
}

Received 20 February 2014; Revised 23 March 2014; Accepted 27 March 2014; Published 13 April 2014

Academic Editor: Toshiaki Kubota

Copyright (c) 2014 Zeynep Alkin et al. This is an open access article distributed under the Creative Commons Attribution License, which permits unrestricted use, distribution, and reproduction in any medium, provided the original work is properly cited.

Background. To investigate the efficacy of selective laser trabeculoplasty (SLT) for lowering intraocular pressure (IOP) in patients with open angle glaucoma (OAG) secondary to emulsified silicone oil (SO). Methodology/Principal Findings. Prospective, interventional, consecutive case series of 11 eyes with sustained elevation of IOP after SO removal. The mean IOP at baseline, week 1 , month 1 , month 3, and month 6 was evaluated. The mean baseline IOP was significantly decreased from $25 \pm 2.7 \mathrm{mmHg}$ to 18.4 $\pm 5.5 \mathrm{mmHg}$ at week $1(P=0.01), 17.9 \pm 3.1 \mathrm{mmHg}$ at month $1(P=0.008), 15.8 \pm 3.9 \mathrm{mmHg}$ at month $3(P=0.003)$, and $16.2 \pm$ $4.7 \mathrm{mmHg}$ at month $6(P=0.004)$. IOP $<21 \mathrm{mmHg}$ was achieved in $91 \%$ of the eyes without a significant complication at month 6 . Conclusion/Significance. SLT may be successful for lowering IOP in patients with OAG secondary to emulsified SO which was not controlled with maximum antiglaucomatous medical treatment.

\section{Introduction}

Silicone oil (SO) as an endotamponade in the treatment of retinal detachments was first introduced by Cibis et al. in 1962 [1]. Along with the rapid development of complicated posterior segment surgeries, the use of $\mathrm{SO}$ has greatly increased [2]. Although SO provides prolonged retinal tamponade and improves the success rate in these challenging cases, it is associated with a high incidence of complications, such as keratopathy, glaucoma, and cataract [3-6].

Glaucoma is one of the most common complications of SO which develops by a variety of pathophysiologic mechanisms including pupillary block, inflammation, synechial angle closure, rubeosis iridis, migration of emulsified, or nonemulsified SO into the anterior chamber. Infiltration and obstruction of the trabecular meshwork directly by emulsified SO are thought to be the driving force in the pathophysiology of SO related secondary open angle glaucoma (OAG) [4-8]. When a secondary glaucoma develops, it is reasonable to proceed with SO removal alone if the retina is completely attached. Even if, SO removal is an effective treatment for lowering intraocular pressure (IOP); it does not always provide IOP control. Success rates of medical treatment in controlling high IOP levels in SO related secondary glaucoma vary between $30 \%$ and $78 \%[9,10]$. While the medical antiglaucomatous treatments have high failure rates in the management of glaucoma secondary to SO, conventional glaucoma surgery has a limited success and carries high risk of complications such as hypotony and phthisis [11, 12].

If angle closure is not a contributing mechanism, selective laser trabeculoplasty (SLT) might be an option for lowering IOP. A great number of studies reported that SLT can be used as an adjunctive therapy to topical glaucoma drops with low complication rates in OAG $[13,14]$. The aim of this study was to investigate the potential efficacy of SLT for lowering the intraocular pressure (IOP) in patients with OAG secondary 
to emulsified SO, in whom IOP could not be controlled with maximum antiglaucomatous medical treatment.

\section{Methods}

This prospective, interventional, single center study enrolled 11 eyes of 11 patients with OAG secondary to emulsified SO. The patients who had previously undergone SO removal were referred to SLT because of uncontrolled IOP with maximum tolerable antiglaucomatous medical treatment between December 2011 and June 2012. All patients had elevated IOP levels at the time of SO removal and were sustained for at least 3 months after removal. The protocol of the study adhered to the tenets of the Declaration of Helsinki. All patients provided informed consent before the procedure. Inclusion criteria were as follows: (1) IOP $\geq 21 \mathrm{mmHg}$ on maximum antiglaucomatous medical treatment; (2) SO droplets in the anterior chamber or anterior chamber angle at the time of removal; (3) an attached retina after removal; (4) an open anterior chamber angle allowing laser application. Exclusion criteria were (1) any type of angle closure glaucoma; (2) an elevated IOP level attributed to previous vitreoretinal surgery other than emulsified SO, such as a scleral buckling procedure; (3) previous laser or surgical glaucoma interventions; (4) the diagnosis of glaucoma antedating vitreoretinal surgery.

Data regarding demographic information, visual acuity, number of glaucoma medications, underlying retinal pathologic findings that required vitreoretinal surgery with SO injection, vitreoretinal surgical procedure, number of vitreoretinal surgeries preceding SO removal, time of SO removal, time elapsed to the first observation of IOP elevation, and status of the lens and anterior chamber angle were recorded. Also parameters and complications of the SLT were recorded.

Preoperative evaluation including measurement of best corrected visual acuity via Snellen chart, measurement of IOP via Goldmann applanation tonometry, gonioscopy, slit lamp biomicroscopy, stereoscopic optic disc evaluation with a 90 diopter lens, and dilated fundus examination with binocular indirect ophthalmoscopy was performed.

2.1. Silicone Oil Removal Method. Silicone oil removal was performed via a pars plana entry. First, an infusion cannula was inserted into the vitreous cavity from an incision at the inferotemporal quadrant $3.0 \mathrm{~mm}$ away from the limbus in aphakic and pseudophakic eyes or $3.5 \mathrm{~mm}$ in phakic eyes. Then, another two pars plana incisions were made at the superotemporal and superonasal quadrants. One incision was used for illuminating the fundus to detect any redetachment at the time of the SO removal. SO was aspirated with a syringe by active aspiration. Then, anterior chamber was washed with a balanced salt solution (BSS Plus, Alcon Laboratories, Fort Worth, Texas, USA) to remove all SO droplets. Incision sites were closed with 7-0 vicryl suture.

2.2. Selective Laser Trabeculoplasty. Immediately before the laser procedure a single application of proparacaine hydrochloride $0.5 \%$ was instilled into the eye. All patients were treated by the same physician (BS). SLT consisted of Nd:YAG laser with a pulse duration of $3 \mathrm{~ns}$ and a spot size of $400 \mu \mathrm{m}$ was performed with the SLT Laserex Tango laser system (Laserex Tango, Ellex Medical, Australia). The energy level ranged from 0.6 to $1.1 \mathrm{~mJ}$. The laser energy was initially set at $0.6 \mathrm{~mJ}$ until a cavitation bubble appeared. Then the laser energy was reduced by $0.1 \mathrm{~mJ}$ increments until no bubble formation was observed; treatment was then continued at this energy level. If no cavitation bubble was observed, the pulse energy was increased by increments of $0.1 \mathrm{~mJ}$ until bubble formation and then decreased as described above. The entire trabecular meshwork which could be seen easily and allowed to laser application was treated with contiguous laser spots. Apraclonidine eye drop was instilled once after SLT. No medical anti-inflammatory treatment applied and all patients continued with the same medical treatment after SLT. Postoperative examination including best corrected Snellen visual acuity, Goldmann applanation tonometry, slit-lamp biomicroscopy, and indirect ophthalmoscopy was performed at week 1 , and at months 1, 3, and 6, postoperatively. Additionally, IOP in the treated eye was measured at first hour following SLT to detect IOP spike of $\geq 5 \mathrm{mmHg}$ from baseline. If so, IOP spike was treated with appropriate antiglaucoma medications. The Wilcoxon signed-rank test was used for comparing pre- and post-SLT IOP measurements.

\section{Results}

Seven $(63.6 \%)$ of the 11 patients were male and $4(36.4 \%)$ were female. The mean age was $38.2 \pm 16.6$ years (range, 19-74 years). The pretreatment clinical characteristics of the patients are listed in Table 1.

The underlying pathology that required vitreoretinal surgery with SO injection was rhegmatogenous retinal detachment with proliferative vitreoretinopathy in 6 eyes, traumatic retinal detachment in 4 eyes, and proliferative diabetic retinopathy with tractional retinal detachment in one eye. All eyes had undergone pars plana vitrectomy with SO injection (1000-centistoke SO in 10 eyes, 5000-centistoke SO in 1 eye), additionally, 3 eyes scleral buckling with encircling, 1 eye lens extraction with phacoemulsification, and 2 eyes pars plana lensectomy at the time of pars plana vitrectomy with SO injection. Inferior peripheral iridectomies were performed at the time of pars plana vitrectomy with SO injection in aphakic eyes ( 2 eyes). All eyes had emulsified SO in the anterior chamber at the time of removal. After SO removal, gonioscopic examination revealed open drainage angle in 10 eyes and open drainage angle with $90^{\circ}$ peripheral anterior synechia located around iridectomy site in one eye (Patient no. 8). We assume that peripheral anterior synechia might have been resulted from iridectomy procedure at the time of pars plana vitrectomy in patient 8 . Furthermore, a few emulsified droplets of SO were identified at the superior anterior chamber angle without obscuring the angle structure on gonioscopy in all eyes (Table 2 summarizes patients' data).

The mean IOP was $25 \pm 2.7 \mathrm{mmHg}$ (range, $21-28 \mathrm{mmHg}$ ) at baseline. After SLT, the mean IOP decreased significantly to $18.4 \pm 5.5 \mathrm{mmHg}$ (range, $12-32 \mathrm{mmHg}$ ) at week 1 , 
TABLE 1: Demographic and clinical characteristics and selective laser trabeculoplasty parameters.

\begin{tabular}{lr}
\hline Variables & Patients $(n=11)$ \\
\hline Age (years), mean \pm SD (range) & $38.2 \pm 16.6(19-74)$ \\
Gender, male/female & $7 / 4$ \\
Number of medications before SLT, mean \pm SD (range) & $2.9 \pm 0.8(2-4)$ \\
Pre-SLT Snellen BCVA (\%) & $1(9 \%)$ \\
$\quad>20 / 200$ & $6(54.6 \%)$ \\
$\quad<20 / 200-C F$ & $4(36.4 \%)$ \\
$\quad \leq$ HM & $25 \pm 2.7(21-28)$ \\
PreSLT IOP (mmHg), mean \pm SD (range) & \\
SLT parameters & \\
$\quad$ Number of laser spots, mean \pm SD (range) & $113 \pm 45(50-160)$ \\
Total energy (mJ), mean \pm SD (range) & $0.82 \pm 0.16(0.7-1.1)$ \\
$\quad$ Degree of angle treated, mean \pm SD (range) & $184 \pm 43(120-270)$ \\
\hline
\end{tabular}

SD: standard deviation; SLT: selective laser trabeculoplasty; BCVA: best corrected visual acuity; CF: counting fingers; HM: hand motion; IOP: intraocular pressure.

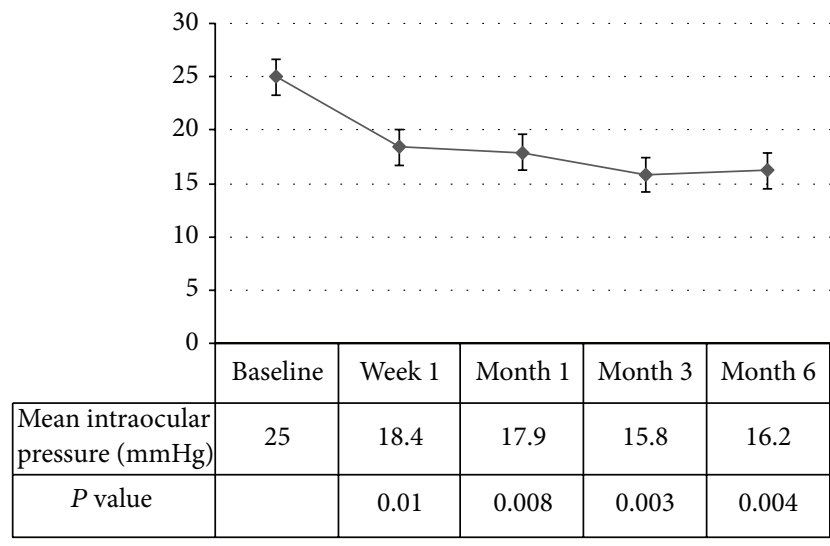

FIGURE 1: The course of mean IOP during the follow-up period.

$17.9 \pm 3.1 \mathrm{mmHg}$ (range, $13-22 \mathrm{mmHg}$ ) at month $1,15.8 \pm$ $3.9 \mathrm{mmHg}$ (range, $10-21 \mathrm{mmHg}$ ) at month 3 , and $16.2 \pm$ $4.7 \mathrm{mmHg}$ (range, $11-24 \mathrm{mmHg}$ ) at month $6(P=0.01$, $0.008,0.003$, and 0.004 , resp.) (Figure 1 ). In one eye, IOP was measured as $32 \mathrm{mmHg}$ at week 1 after SLT and returned to normal values with oral acetazolamide therapy for 3 days (Patient no. 5). IOP level of $<21 \mathrm{mmHg}$ at 6 months of therapy was achieved in ten eyes (91\%). Pressure spike at the first hour following SLT was detected in one eye. Transient inflammatory reaction $(1+$ cells $)$ was found in 2 eyes.

\section{Discussion}

Glaucoma is not a rare complication of retinal detachment surgery with the incidence of $6 \%-56 \%$. Mechanisms of glaucoma with SO include pupillary block, inflammation, synechial angle closure, rubeosis iridis, migration of emulsified or nonemulsified SO into the anterior chamber, and idiopathic open-angle glaucoma [4-8]. Emulsification of SO carries a high risk of IOP elevation by mechanically obstructing the trabecular meshwork [15]. It has been confirmed by pathologic examination that emulsified SO drops or macrophages loaded with SO drops can block the trabecular meshwork. Elevated IOP is mainly dependent on the blockage of aqueous outflow [16]. Leaver et al. noted emulsified SO in 9 of 14 eyes showing elevated IOP after pars plana vitrectomy with SO injection. They reported histopathologic evidence of the presence of macrophages loaded with SO within the trabecular meshwork without evidence of structural damage to the collagen fibers and the trabecular endothelium [17]. In our study, the gonioscopic evaluation showed that the anterior chamber angle was open and no synechia was observed (except patient no. 8 with partial peripheral anterior synechia). We suppose that the elevated IOP mainly dependent on the blockage of outflow of aqueous humor by emulsified SO.

The benefit of early SO removal before the emulsification was demonstrated to be effective for IOP regulation in higher proportion of the eyes [18]. However, the removal of emulsified SO does not necessarily prevent the development of glaucoma. Flaxel et al. reported that elevated IOP persisted in all eyes with secondary glaucoma after SO removal [19]. Furthermore, SO removal itself can cause IOP elevation by splitting SO droplets into smaller bubbles which are more likely to obstruct trabecular meshwork during SO removal [20]. In the study by Wei et al., IOP was demonstrated to be elevated postoperatively in 11 of 64 cases after SO removal [21]. The incidence of glaucoma was reported to be $4.7 \%$ after SO removal in a study by Casswell and Gregor [22]. Lin et al. suggested that anterior chamber irrigation is important to remove all SO droplets to avoid IOP elevation secondary to emulsified SO [20]. Irrigation of anterior chamber as much as possible may be helpful to remove SO droplets; however, it is difficult to remove all retained $\mathrm{SO}$ droplets in the trabecular meshwork and to control the elevated IOP with this maneuver as in our patients. Moisseiev et al. performed anterior chamber wash-out for several times in an attempt to remove residual SO droplets and to achieve better IOP control after SO removal, but the method was not found to be effective in $91 \%$ of the eyes [18]. 


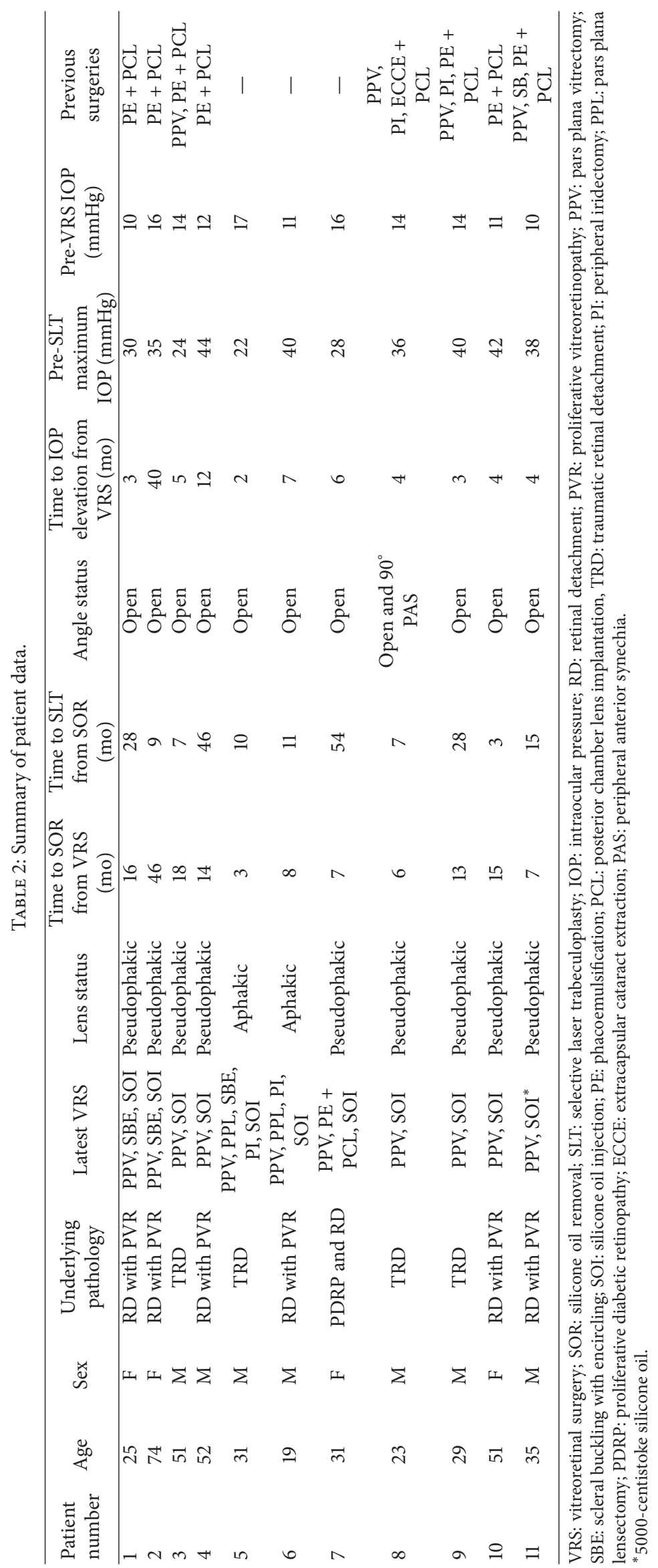


Treatment of patients with glaucoma secondary to SO is still controversial. In the case of glaucoma secondary to $\mathrm{SO}$, aqueous suppressants should be the first line therapy to lower the pressure [23]. However, the ability to control IOP with glaucoma medications shows variable efficacy, and success rates vary between $30 \%$ and $78 \%[9,10]$. On the other hand, traditional filtering surgery is technically difficult because of the conjunctival scarring that results from the multiple retinal surgeries and carries a poor prognosis [9]. Glaucoma drainage implants may be another surgical option in the patients with glaucoma secondary to SO; however, there is a possibility of SO escape via the drainage tube [24]. Successful results with cyclodestructive procedures for lowering IOP have been reported in $74 \%$ to $82 \%$ of patients with medically uncontrolled glaucoma secondary to SO at one year. Unfortunately, multiple treatments may be required to obtain pressure control and visual loss is not uncommon after cyclophotocoagulation. For this reason, cyclodestructive procedures are less desirable treatment options in such patients $[11,25,26]$.

Previous studies verified that SLT was safe and effective for lowering IOP in patients with OAG [13, 14, 27, 28]. SLT uses the $532 \mathrm{~nm}$ laser targeting the trabecular meshwork where the most resistance to aqueous outflow exists [27]. Several experimental studies using SLT have shown a release of chemotactic and vasoactive agents. These cytokines cause remodeling of the extracellular matrix and are involved in stimulation of cellular activity by increasing the recruitment and number of macrophages $[27,29]$. We propose that activating the macrophages loaded with $\mathrm{SO}$ and remodeling the extracellular matrix in the trabecular meshwork by laser application may lead to increased aqueous outflow in glaucoma secondary to emulsified SO as in other types of OAGs. Latina and De Leon found that 40 eyes (64.5\%) which were on maximum tolerable medical therapy achieved an IOP reduction of $\geq 20 \%$ from baseline with 360 degree SLT at month 12 [27]. Lowering the IOP $<21 \mathrm{mmHg}$ was achieved in $91 \%$ of eyes without a significant complication in the present study. This difference in the success rates between the studies can be explained by the differences in success criteria, clinical characteristics of the patients, and duration of follow-up time.

A logical approach for the management of patients with glaucoma secondary to SO should be modified according to the individual clinical presentation. When emulsified SO blocks the trabecular meshwork, it is reasonable to proceed with SO removal and topical antiglaucomatous treatment, if the retina is completely attached. We suggest that SLT may be a treatment option for the patients with OAG secondary to emulsified SO which are not at high risk for progressive glaucomatous damage to save time before more invasive surgical interventions are performed.

The limitations of this study were the lack of a control group, limited number of the patients, and short follow-up period. Despite limitations, as a proof of principle pilot study, it may raise possibilities of further investigations on the role of IOP lowering potential of SLT in these challenging cases of glaucoma.

In conclusion, this study shows that SLT is a safe and effective method for lowering IOP in OAG secondary to emulsified SO. To our knowledge, this is the first report of safety and efficacy of SLT as an adjunctive therapy for emulsified SO related glaucoma. Although early results of this study are encouraging, long-term outcomes are needed to determine the efficacy of this therapy. Further investigations on a larger number of eyes are necessary to determine the efficacy of SLT in the patients who suffered from glaucoma secondary to emulsified SO.

\section{Conflict of Interests}

The authors declare that there is no conflict of interests regarding the publication of this paper.

\section{Authors' Contribution}

Zeynep Alkin contributed to drafting the paper. Banu Satana and Abdullah Ozkaya contributed to conception and design of the paper. Berna Basarir and Cigdem Altan contributed to acquisition of data. Zeynep Alkin and Banu Satana contributed to analysis and interpretation of data. Ahmet Taylan Yazici and Ahmet Demirok gave final approval of the version to be published.

\section{References}

[1] P. A. Cibis, B. Becker, E. Okun, and S. Cowan, "The use of liquid silicone in retinal detachment surgery," Archives of Ophthalmology, vol. 68, pp. 590-599, 1962.

[2] R. Machemer, "Proliferative vitreoretinopathy (PVR): a personal account of its pathogenesis and treatment. Proctor lecture," Investigative Ophthalmology and Visual Science, vol. 29, no. 12, pp. 1771-1783, 1988.

[3] G. W. Abrams, S. P. Azen, C. C. Barr et al., "The incidence of corneal abnormalities in the silicone study: Silicone Study Report 7," Archives of Ophthalmology, vol. 113, no. 6, pp. 764769,1995

[4] J. L. Federman and H. D. Schubert, "Complications associated with the use of silicone oil in 150 eyes after retina-vitreous surgery," Ophthalmology, vol. 95, no. 7, pp. 870-876, 1988.

[5] J. H. Yeo, B. M. Glaser, and R. G. Michels, "Silicone oil in the treatment of complicated retinal detachments," Ophthalmology, vol. 94, no. 9, pp. 1109-1113, 1987.

[6] C. C. Barr, M. Y. Lai, J. S. Lean et al., "Postoperative intraocular pressure abnormalities in the Silicone Study: Silicone Study Report 4," Ophthalmology, vol. 100, no. 11, pp. 1629-1635, 1993.

[7] J. D. Henderer, D. L. Budenz, H. W. Flynn Jr., J. C. Schiffman, W. J. Feuer, and T. G. Murray, "Elevated intraocular pressure and hypotony following silicone oil retinal tamponade for complex retinal detachment: incidence and risk factors," Archives of Ophthalmology, vol. 117, no. 2, pp. 189-195, 1999.

[8] L. R. de Corral, S. B. Cohen, and G. A. Peyman, "Effect of intravitreal silicone oil on intraocular pressure," Ophthalmic Surgery, vol. 18, no. 6, pp. 446-449, 1987.

[9] Q. H. Nguyen, M. A. Lloyd, D. K. Heuer et al., "Incidence and management of glaucoma after intravitreal silicone oil injection for complicated retinal detachments," Ophthalmology, vol. 99, no. 10, pp. 1520-1526, 1992.

[10] S. G. Honavar, M. Goyal, A. B. Majji, P. K. Sen, T. Naduvilath, and L. Dandona, "Glaucoma after pars plana vitrectomy and 
silicone oil injection for complicated retinal detachments," Ophthalmology, vol. 106, no. 1, pp. 169-177, 1999.

[11] T. C. Chen, L. R. Pasquale, D. S. Walton, and C. L. Grosskreutz, "Diode laser transscleral cyclophotocoagulation," International Ophthalmology Clinics, vol. 39, no. 1, pp. 169-176, 1999.

[12] D. L. Budenz, K. E. Taba, W. J. Feuer et al., "Surgical management of secondary glaucoma after pars plana vitrectomy and silicone oil injection for complex retinal detachment," Ophthalmology, vol. 108, no. 9, pp. 1628-1632, 2001.

[13] I. McIlraith, M. Strasfeld, G. Colev, and C. M. L. Hutnik, "Selective laser trabeculoplasty as initial and adjunctive treatment for open-angle glaucoma," Journal of Glaucoma, vol. 15, no. 2, pp. 124-130, 2006.

[14] T. Gračner, "Intraocular pressure response of capsular glaucoma and primary open-angle glaucoma to selective Nd:YAG laser trabeculoplasty: a prospective, comparative clinical trial," European Journal of Ophthalmology, vol. 12, no. 4, pp. 287-292, 2002.

[15] K. G. Riedel, V.-P. Gabel, L. Neubauer, A. Kampik, and O.E. Lund, "Intravitreal silicone oil injection: complications and treatment of 415 consecutive patients," Graefe's Archive for Clinical and Experimental Ophthalmology, vol. 228, no. 1, pp. 1923, 1990.

[16] W. R. Lee, "Doyne lecture: the pathology of the outflow system in primary and secondary glaucoma," Eye, vol. 9, no. 1, pp. 1-23, 1995.

[17] P. K. Leaver, R. H. B. Grey, and A. Garner, "Silicone oil injection in the treatment of massive preretinal retraction. II. Late complications in 93 eyes," British Journal of Ophthalmology, vol. 63 , no. 5, pp. 361-367, 1979.

[18] J. Moisseiev, A. Barak, T. Manaim, and G. Treister, "Removal of silicone oil in the management of glaucoma in eyes with emulsified silicone," Retina, vol. 13, no. 4, pp. 290-295, 1993.

[19] C. J. Flaxel, S. M. Mitchell, and G. W. Aylward, "Visual outcome after silicone oil removal and recurrent retinal detachment repair," Eye, vol. 14, no. 6, pp. 834-838, 2000.

[20] X. F. Lin, L. Y. Liang, M. K. Lin, and Z. H. Yuan, "Treatment of glaucoma secondary to silicone oil retention," Retina, vol. 25, no. 4, pp. 515-517, 2005.

[21] W. B. Wei, C. L. Monin, and H. Hamard, "The effects of silicone oil removal on the complications after retinal detachment surgery," Chinese Journal of Ocular Fundus Diseases, vol. 13, pp. 22-23, 1997.

[22] A. G. Casswell and Z. J. Gregor, "Silicone oil removal. II. Operative and postoperative complications," British Journal of Ophthalmology, vol. 71, no. 12, pp. 898-902, 1987.

[23] D. Minckler, S. Best, S. Vernon, and J. M. Liebmann, "Silicone oil glaucoma," Journal of Glaucoma, vol. 10, no. 1, pp. 51-54, 2001.

[24] P. Senn, E. R. Buchi, B. Daicker, and I. Schipper, "Bubbles in the bleb-troubles in the bleb? Molteno implant and intraocular tamponade with silicone oil in an aphakic patient," Ophthalmic Surgery, vol. 25, no. 6, pp. 379-382, 1994.

[25] P. A. Bloom, J. C. Tsai, K. Sharma et al., "Cyclodiode': transscleral diode laser cyclophotocoagulation in the treatment of advanced refractory glaucoma," Ophthalmology, vol. 104, no. 9, pp. 1508-1520, 1997.

[26] S. K. Han, K. H. Park, D. M. Kim, and B. L. Chang, "Effect of diode laser trans-scleral cyclophotocoagulation in the management of glaucoma after intravitreal silicone oil injection for complicated retinal detachments," British Journal of Ophthalmology, vol. 83, no. 6, pp. 713-717, 1999.
[27] M. A. Latina and J. M. S. De Leon, "Selective laser trabeculoplasty," Ophthalmology Clinics of North America, vol. 18, no. 3, pp. 409-419, 2005.

[28] N. Sayin, Z. Alkin, A. Ozkaya et al., "Efficacy of Selective Laser Trabeculoplasty in medically uncontrolled glaucoma," ISRN Ophthalmology, vol. 2013, Article ID 975281, 4 pages, 2013.

[29] T. R. Kramer and R. J. Noecker, "Comparison of the morphologic changes after selective laser trabeculoplasty and argon laser trabeculoplasty in human eye bank eyes," Ophthalmology, vol. 108, no. 4, pp. 773-779, 2001. 


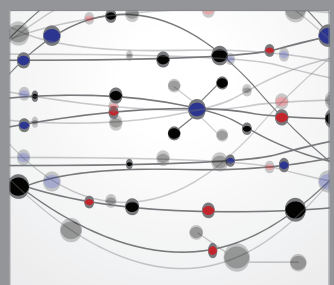

The Scientific World Journal
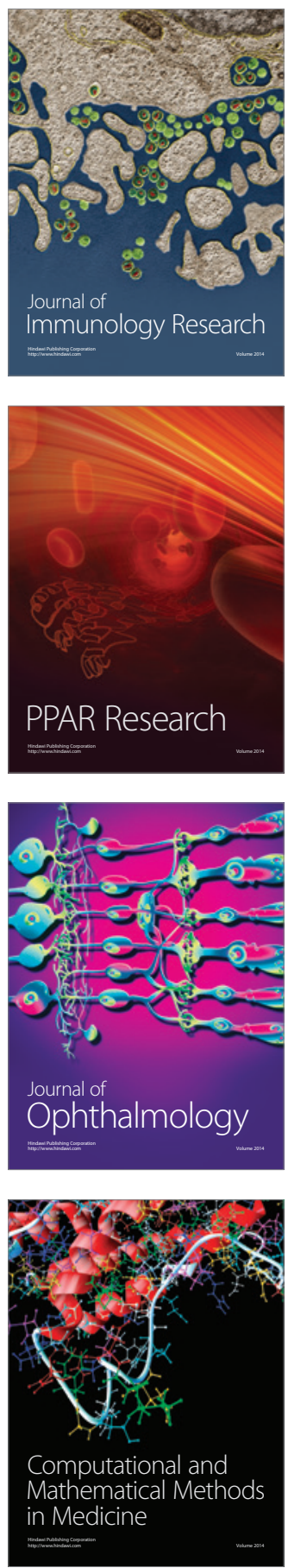

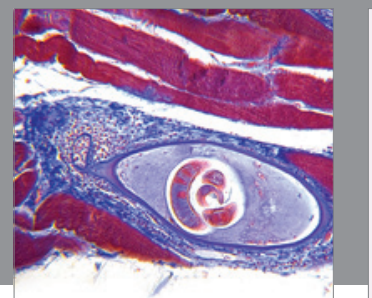

Gastroenterology

Research and Practice
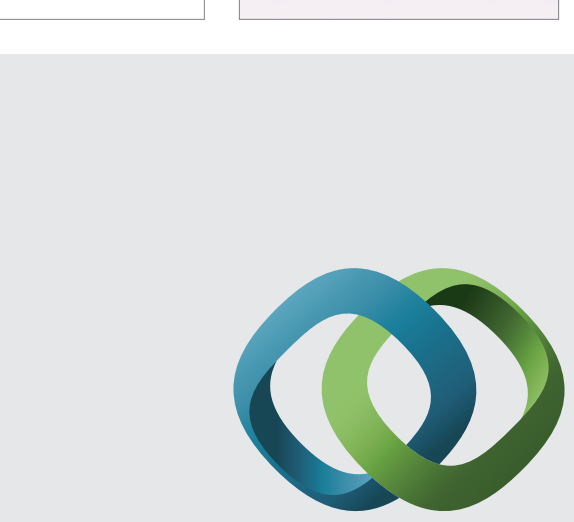

\section{Hindawi}

Submit your manuscripts at

http://www.hindawi.com
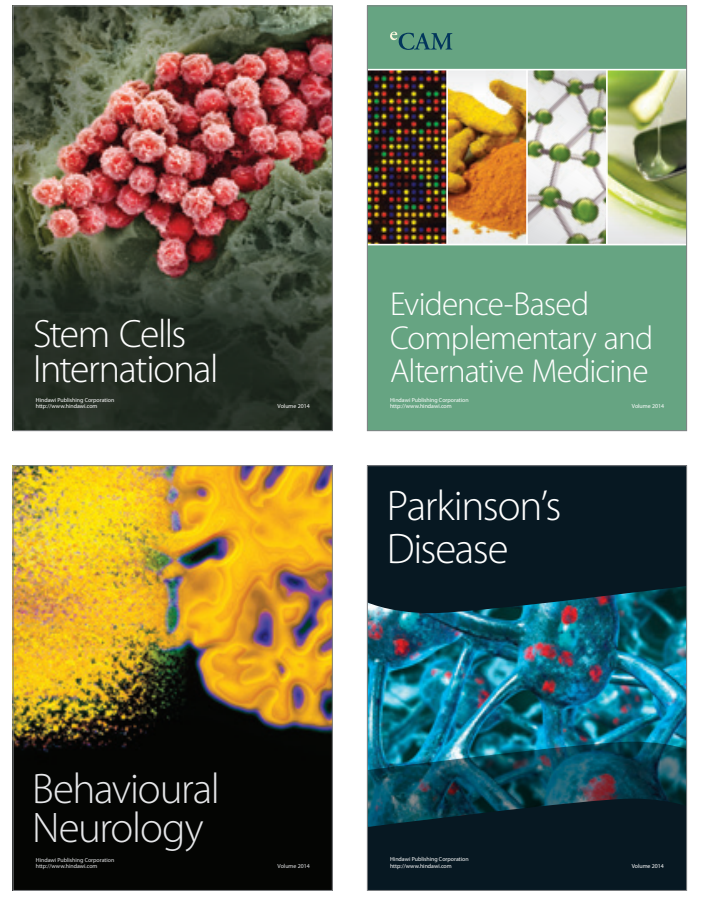
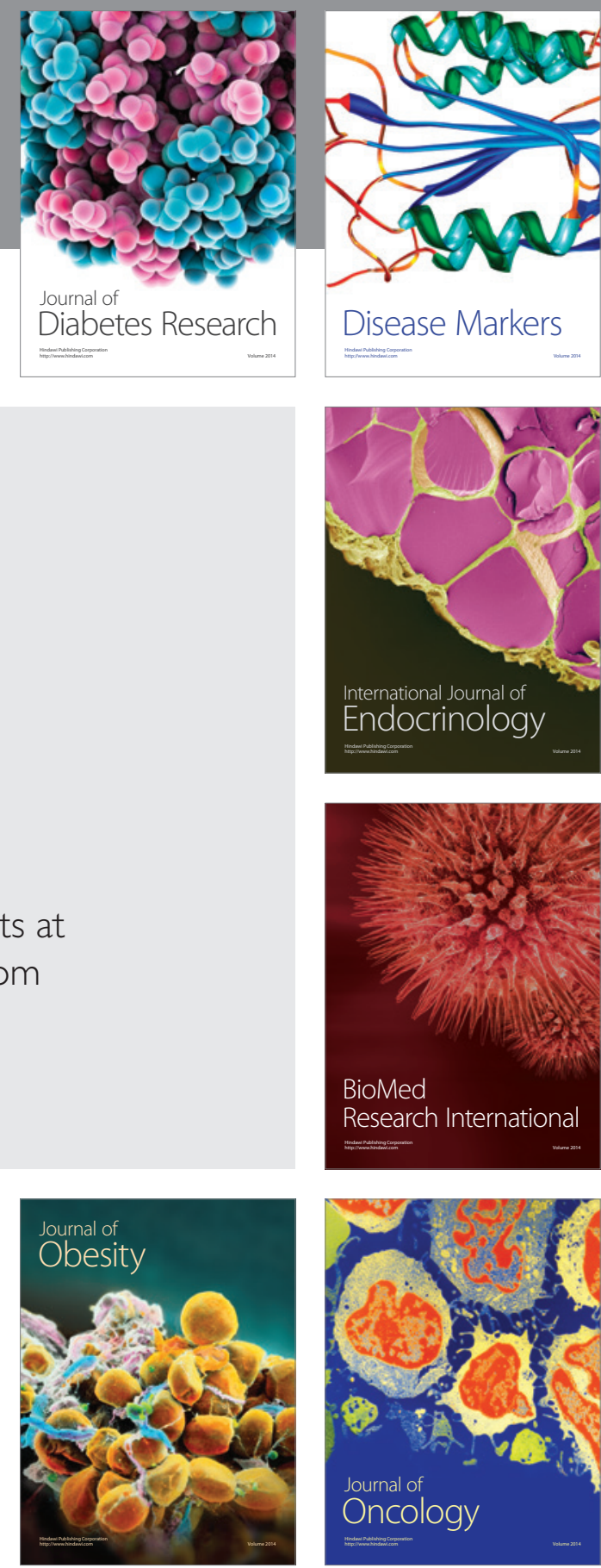

Disease Markers
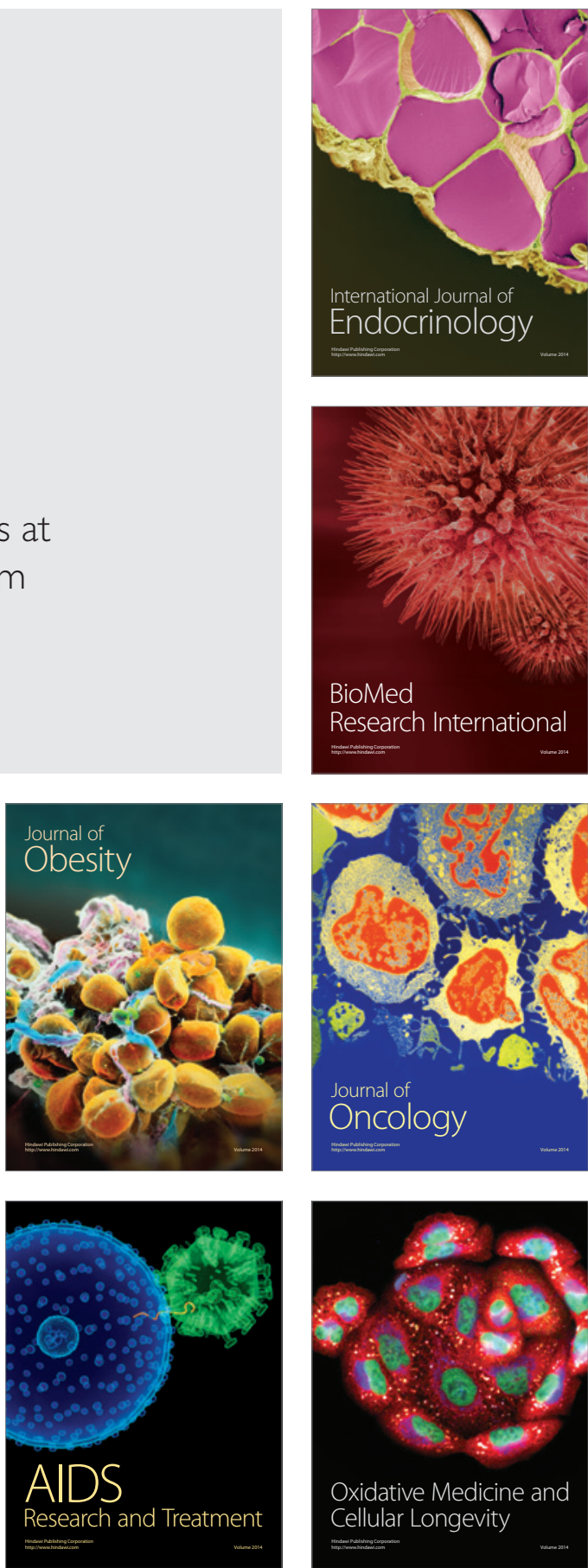